\title{
Aerodynamic efficacy of adding yaw-wise rotational degree of freedom to an airplane flap
}

\author{
Kazuhisa Chiba ${ }^{1}$ (D) Tatsuro Komatsu ${ }^{1} \cdot$ Takeshi Ito $^{2}$
}

Received: 22 May 2020 / Revised: 23 July 2020 / Accepted: 12 August 2020 / Published online: 24 August 2020

(C) The Author(s) 2020

\begin{abstract}
This study scrutinized the aerodynamic change of adding a yaw-wise rotational degree of freedom to a single slotted flap of airplane via computational fluid dynamic analyses. Existing slotted flaps have spanwise constant gaps and are disharmonious with the 3D nature of the flow field. A flap geometry and its angle condition are sensitive to aerodynamic performance; small variations in them must be useful in aerodynamic improvement. To add the yaw-wise rotation to a flap is a lower hurdle than to attain other high-lift systems. Therefore, after defining a simple configuration consisting of a fuselage, a wing, and a single slotted flap, we investigated the mesh dependency to consider the diversity in flow phenomena precisely; we examined the effect of the yaw-wise rotation for the flap on improving the whole lift. To place the flap at suitable yaw-wise rotation angles consequently effected raising the lift. We revealed the physical mechanism that accelerating the fluid in the gap between the wing and the flap changes the separation structure on the flap upper surface and grows the lift.
\end{abstract}

Keywords Airplane flap - Aerodynamic performance $\cdot$ Yaw-wise rotational degree of freedom - Computational fluid dynamics

\section{Introduction}

A high-lift device (HLD) is a movable mechanism attached to the wing of an airplane [5]. The demand for gaining airplane speed has optimized airplane geometry to fit the transonic cruise, which is the longest in a flight profile [6]. As a result, as the required lift for subsonic takeoff/landing was deficient, airplanes devised high-lift systems to compensate for this. Modern planes have different geometry design strategies between transonic cruising and subsonic takeoff/landing conditions to accomplish higher speeds. Although to raise the angle of attack enables growing the lift, there is the upper limit due to stall. So, high-lift systems are vital mechanisms not only for prevailing airplanes, but also for next-generation ones, which will speed up in the future.

There are three principal types of high-lift systems: the flap mechanism, the boundary-layer control mechanism, and

Kazuhisa Chiba

kazchiba@uec.ac.jp

1 The University of Electro-Communications, 1-5-1, Chofugaoka, Chofu, Tokyo 182-8585, Japan

2 Japan Aerospace Exploration Agency, 7-44-1, Jindaiji-higashi, Chofu, Tokyo 182-8522, Japan the turbulence utilization mechanism [5]. Estimating the aerodynamic performance of high-lift systems is still a challenge in airplane design due to the complexity of the fluid phenomenon [41]. With the progress of schemes on computational fluid dynamics (CFD) and skills of wind tunnel tests, this study would concentrate on the flap mechanism. We are deepening the perception of knowledge via the American Institute of Aeronautics and Astronautics (AIAA) high-lift prediction workshops [36-38]. There are studies on alternative methods of HLDs based on perceiving the aerodynamic principle of HLDs [41], such as active control of the fluid by moving the flap position [31], using plasma actuators [22], and placing vortex generators [21]. However, they have not yet attained any alternative ways to practical use. A flap mechanism is still a viable option in terms of practicality.

We can generally classify the flap mechanism into two kinds of flaps: leading-edge and trailing-edge flaps, and further subdivide them [5]. Airplane retracts a leading-edge flap into the leading edge of the wing during a cruise; a leading-edge flap shifts the separation on the wing backward by circumventing the airflow on the leading-edge flap lower surface into the gap between the leading-edge flap and the wing. It delays the stall and raises the lift [5]. A trailing-edge flap, which is put away in the trailing edge 
of the wing during a cruise, yields a cambering effect and diminishes the static pressure near the wing's trailing edge. It also provides the airflow from the wing underside to the trailing-edge flap upside to prevent the separation on the trailing-edge flap upper surface. These facilitate the rise in the lift [5]. A trailing-edge flap likewise acts as an aerobrake under landing conditions. Indeed, a wide range of airplanes accepts the trailing-edge flap, from small ones with propeller propulsion systems [39] to medium/large-sized bodies with jet engines [32] and even fighters [29]. The use of a leadingedge flap and a trailing-edge flap together generally improves takeoff/landing aerodynamic performance [5]. However, this study centers on only a trailing-edge flap to isolate the influence on each other. Henceforth, we write a trailing-edge flap as merely a flap.

A typical HLD used in modern commercial airplanes is a slotted flap [44], which forms a gap between a wing and a flap [1]. This mechanism has all the effects of a trailing-edge flap. However, a single slotted flap has a translational degreeof-freedom mechanism that moves along flap tracks [35]. Hence, the spanwise gap between the wing and the flap is invariable; the slotted flap cannot cope with the 3D nature of the flow structure that arose on/over the flap upside. Flexible mechanisms, such as morphing [7,30] and dispersive [34] flaps, have been proposed to solve this issue. Nevertheless, they are distinct from contemporary devices that produce static gaps in the spanwise direction.

In a comparable concept, we advance the idea of changing the spanwise gap by appending a degree of yaw-wise rotation for a single slotted flap, which has only a degree of freedom of translation along flap tracks. We anticipate this idea involves prompt feasibility because it does not need any additional elements to airplanes currently in operation. This study examines the aerodynamic significance on a variable spanwise gap between a wing and a single slotted flap by pursuing the feasibility of a simple system that is an extension of popular configurations.

This paper is organized as follows. Section 2 concisely explains the problem definition and the CFD methods to be used. In Sect. 3, to arrange the surface mesh density when analyzing the object, we investigate the dependency of it. Section 4 discusses the aerodynamic benefit and the diversity on the flow structure that the yaw-wise rotation to the flap yields. Section 5 concludes this study.

\section{Problem definition}

\subsection{Configuration}

Since this study aims at scrutinizing the availability of the idea to add a yaw-wise rotation to a single slotted flap, we define the object model by a simple configuration consisting

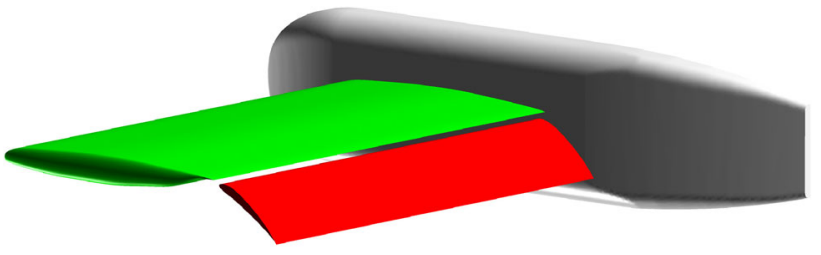

(a) bird's eye view



(b) side view

Fig. 1 Configuration of the computational model consisting of a fuselage, a wing, and a single slotted flap. This figure visualizes the flap at the neutral condition without any yaw-wise rotation

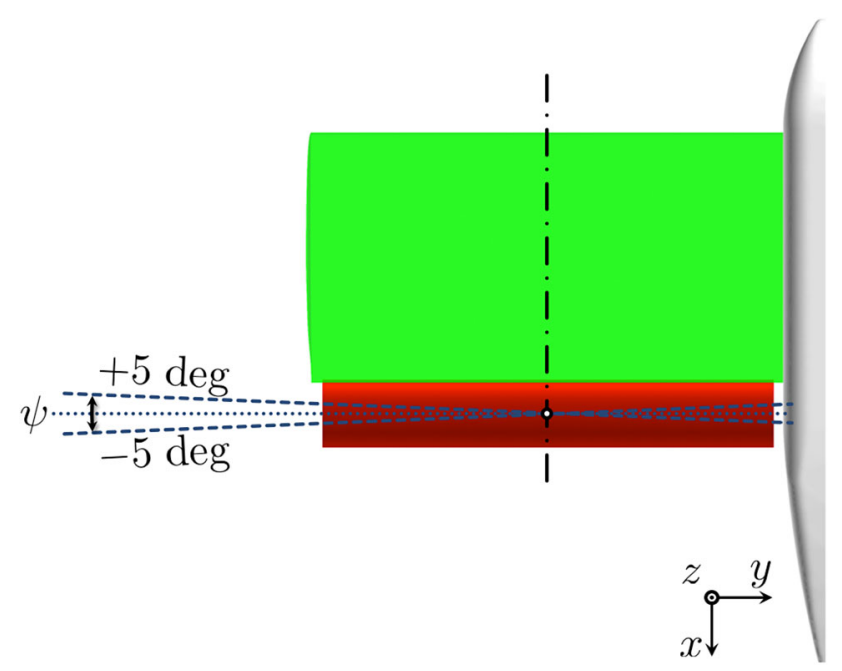

Fig. 2 The definition of yaw-wise rotation angle $\psi$ and its acceptable range on top view. The figure depicts the wing translucently to see the overlap of the wing for the flap

only of a fuselage, a wing, and a single slotted flap shown in Fig. 1. We decide each component as follows.

Wing: our wing conforms to the main wing of "OTOMO" [26]; Japan Aerospace Exploration Agency (JAXA) designed it for researching noise reduction. The main wing is a rectangular planform for simplification. The cross-sectional airfoil shape of the main wing originates in the JAXA standard model [45]. We round the wingtip to alleviate occurring any wingtip vortices.

Flap: we emulate the flap geometry of OTOMO according to the wing. The span length of the flap is $95 \%$ of that of the wing; the flap root/tip shortens by $2.5 \%$. These are because the flap root with yaw-wise rotation does not interfere with the fuselage and the wing (the flap tip does not interfere with the wing); we retain the flap's spanwise symmetry. Besides, the flap root/tip is cleanly cut on a flat surface. Note that we fix the flap setting for the wing at the neutral condition without 
yaw-wise rotation: the flap angle of $35.0^{\circ}$, the gap/overlap of $0.0166 c / 0.01 c$ between the wing and the flap, where $c$ denotes the chord length of the wing. These position parameters come from the landing condition of OTOMO.

We placed the yaw-wise rotational center of the flap at the intersection of the rectangular diagonals when projected on the $x-y$ plane; it rotates around the $z$-axis. We assume the clockwise direction is positive when viewed from the $+z$ direction in the $-y$ region, as shown in Fig. 2. This study will examine five cases: $\psi= \pm 2.5^{\circ}, \pm 5.0^{\circ}$, and the neutral reference condition of the yaw-wise rotational angle $\psi$ of $0.0^{\circ}$.

Fuselage: we employ the fuselage geometry of NASA's trapezoidal wing configuration used in the first AIAA CFD high-lift prediction workshop [37] and utilize computeraided design data, which the workshop released on the website. $^{1}$

\subsection{Geometry and mesh generations}

OpenVSP [8] creates the geometry, then Dassault Systèmes SOLIDWORKS modifies it minutely. MEGG3D [10-20] generates an unstructured surface mesh. Because of not considering the crosswind to the body, we make a half model. The computational space is a hemisphere; we fix the diameter of its outer boundary to be 40 times the fuselage length. Then, MEGG3D creates a hybrid volume mesh within the above computational space. To resolve boundary layers precisely [25], we apiece set the maximum number of prism layers, the thickness of its first layer, and the stretching factor for tetrahedra/prism layers to be $50,(100 \sqrt{R e})^{-1}$, and $1.05 / 1.12$, where $R e$ denotes the Reynolds number.

\subsection{Flow solver}

We use a compressible flow solver TAS [24,27], which solves the 3D Raynolds-averaged Navier-Stokes equations by an unstructured MUSCL-type cell-vertex finite volume method [2] with Venkatakrishnan's differentiable limiter [43] for keeping second-order spatial accuracy. TAS utilizes schemes: Harten-Lax-van Leer-Einfeldt-Wada method [28] for numerical flux computations; lower-upper symmetric Gauss-Seidel implicit method [40] for time integration. Japanese aircraft design has widely employed TAS, such as Mitsubishi SpaceJet [42] and JAXA's Silent Supersonic Technology Demonstrator [4]; we have sophisticated it by contrasting with wind tunnel test results [9].

The variable $\chi$ used in the above MUSCL scheme sets 0.5 [24] to alleviate mathematical error. The limiter has a

\footnotetext{
1 "1st AIAA CFD High Lift Prediction Workshop-Geometries" available online at https://hiliftpw.larc.nasa.gov/workshop1/geometries. html [retrieved 20 February, 2018].
}

Table 1 The number of cells/nodes of meshes

\begin{tabular}{lll}
\hline $\begin{array}{l}\text { Mesh } \\
\text { density }\end{array}$ & $\begin{array}{l}\text { Surface cells } \\
\text { (million) }\end{array}$ & $\begin{array}{l}\text { Number of } \\
\text { nodes (million) }\end{array}$ \\
\hline Coarse & 0.05 & 2.2 \\
Medium & 0.16 & 5.5 \\
Fine & 0.41 & 15.5 \\
\hline
\end{tabular}

bias parameter $K$ for stable convergence, which contains the extremely high sensitivity of the limiter. It also copes with precisely computing the numerical dissipation and predicted drag when the limiter has a small number. This study adopts Menter's shear stress transport two-equation turbulence model [23]. Note that TAS modifies the production term to

$\tau_{i j} \frac{\partial u_{i}}{\partial x_{j}}=\mu_{T} \Omega^{2}$

for grasping vortex generations in the subsonic speed correctly. Computations run on the part of the cluster with HP ProLiant SL250s G8 with Intel Xeon E5-2670 2.60 GHz in Information Technology Center, the University of ElectroCommunications.

\subsection{Computational conditions}

We set the angle of attack $\alpha$, which the wing stipulates, $0.0^{\circ}$ to direct toward the flap lift, and alleviate the impact of the wing lift as much as possible. $R e$ in the wing chord length is $4.3 \times 10^{6}$. We fix Mach number $M$ to 0.2 according to the OTOMO's landing condition. Since we ignore crosswind computational requirements this time, the $y$ and $z$ components of the inflow are zero. Thus, we suppose computing with the half model symmetrically.

\section{Mesh dependency}

We determine mesh density for accurate analyses with three different meshes. We pursue it under the $\psi=-5.0^{\circ}$ condition because of the most substantial change in the spanwise direction of the gap between the wing and the flap. We will examine the variations in aerodynamic performance and flow structure.

We name three types of meshes: "Coarse", "Medium", and "Fine"; Table 1 arranges the number of cells/nodes in each mesh. Figures 3 and 4 display representative views of each surface/volume mesh. Note that we modify the surface mesh density and make the volume mesh in the similar parameters between these three types. Thus, the difference in the number of cells/nodes between the three meshes depends 

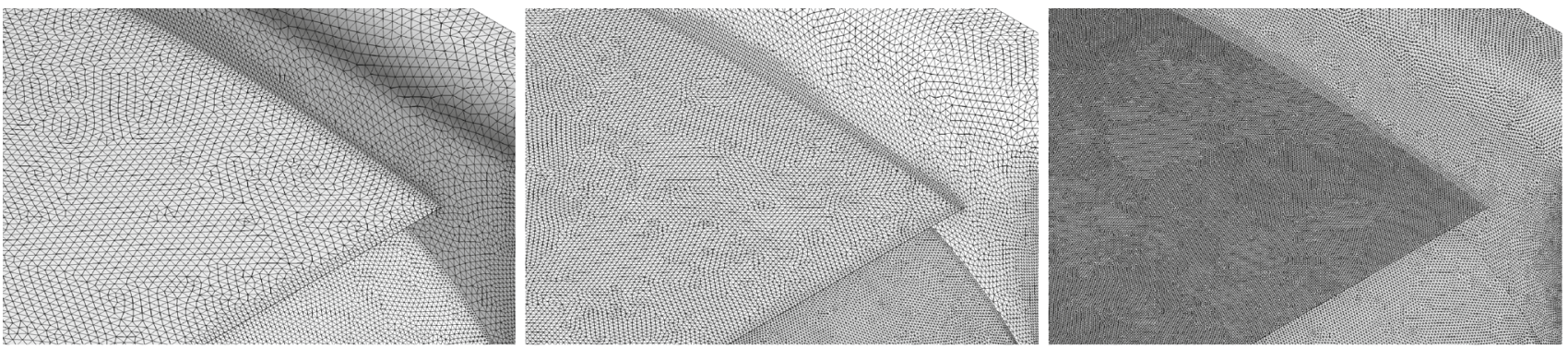

(a) root location of the wing


(b) tip location of the wing

Fig. 3 Close-up view of the surface meshes near the wing between Coarse (left), Medium (center), and Fine (right)

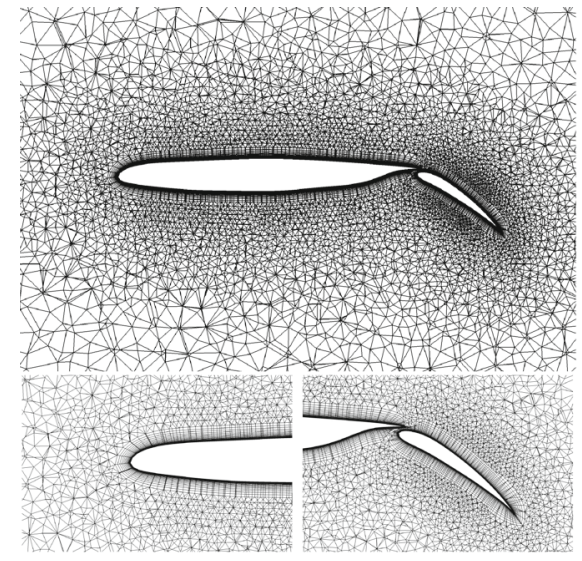

(a) Coarse

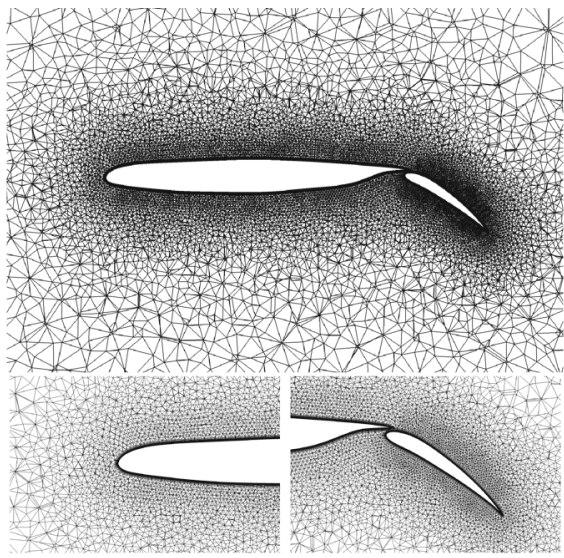

(b) Medium

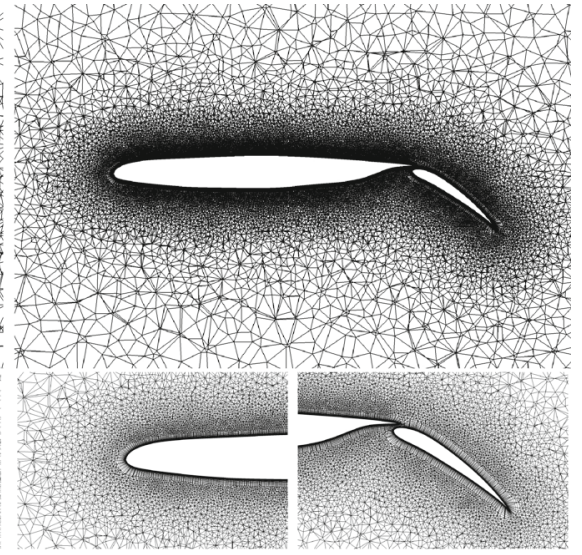

(c) Fine

Fig. 4 Cross-sectional views of the volume meshes at the 50\% spanwise position: overview and enlarged views around the wing leading edge and the flap

on that of surface cells roughly. We rule convergence as $\Delta C_{\mathrm{D}} \leq 1 \mathrm{cnt}$; the wall-clock run time to convergence takes about 70/160/500 h on the Coarse/Medium/Fine mesh using 640 cores of the cluster.

Figure 5 compiles the transition of the lift coefficient $C_{\mathrm{L}}$ and the drag coefficient $C_{\mathrm{D}}$ values by each mesh. $N$ on the horizontal axis represents the total number of nodes; $N^{-2 / 3}$ is the function provided for the second-order spatial accuracy based on Richardson's extrapolation [33]. Also, to pursue the cause of the discrepancy in the $C_{\mathrm{L}}$ and the $C_{\mathrm{D}}$, Fig. 6 depicts the respective pressure coefficient $C_{\mathrm{p}}$ surface distribution and its streamline. When we describe detailed comparisons in the following subsections and state the conclusion earlier, although the Coarse mesh misses the separation on the wing undersurface near the root, the Medium and Fine meshes grasp it. Thus, we utilize medium-density meshes in Sect. 4 due to the computational cost.

\section{$3.1 C_{\mathrm{L}}$}

Figure 5a declares that the mesh convergence of the $C_{L}$ is adequate because there is only a difference of at most $0.5 \%$ $C_{\mathrm{L}}$ between mesh densities. The contrast of the $C_{L}$ by the fuselage is negligible. The wing and the flap affect an equivalent degree. Figure 6 implies that the computational variation of the interference between the wing and the flap brings the $C_{L}$ fluctuation. Figure $6 \mathrm{~b}$ and $\mathrm{c}$ indicates that the Medium and Fine meshes catch the separation around the root trailing edge of the wing underside where the flap overlaps; they exhibit a comparable surface-streamline pattern. 


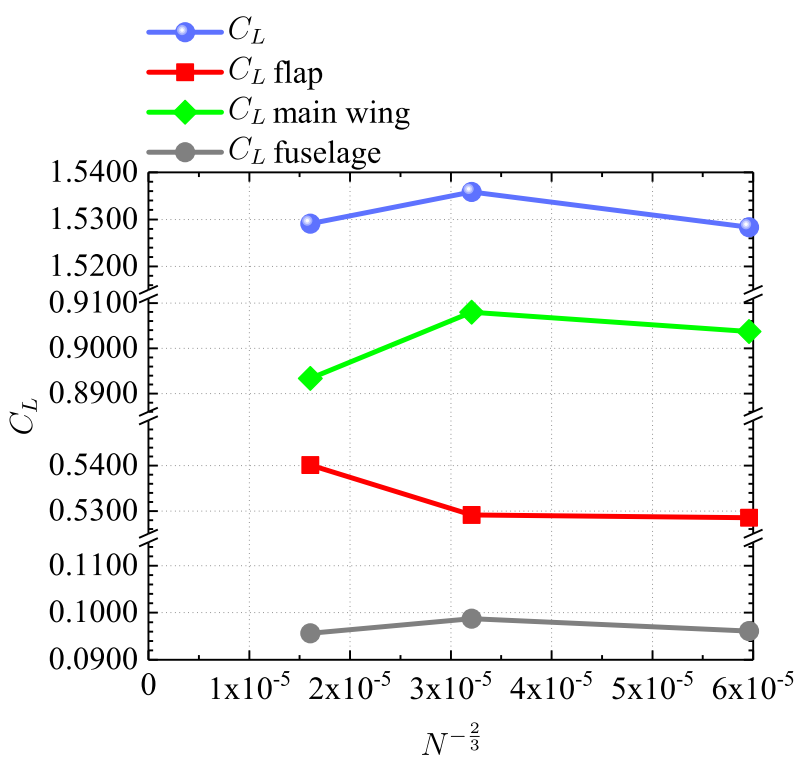

(a) $C_{L}$

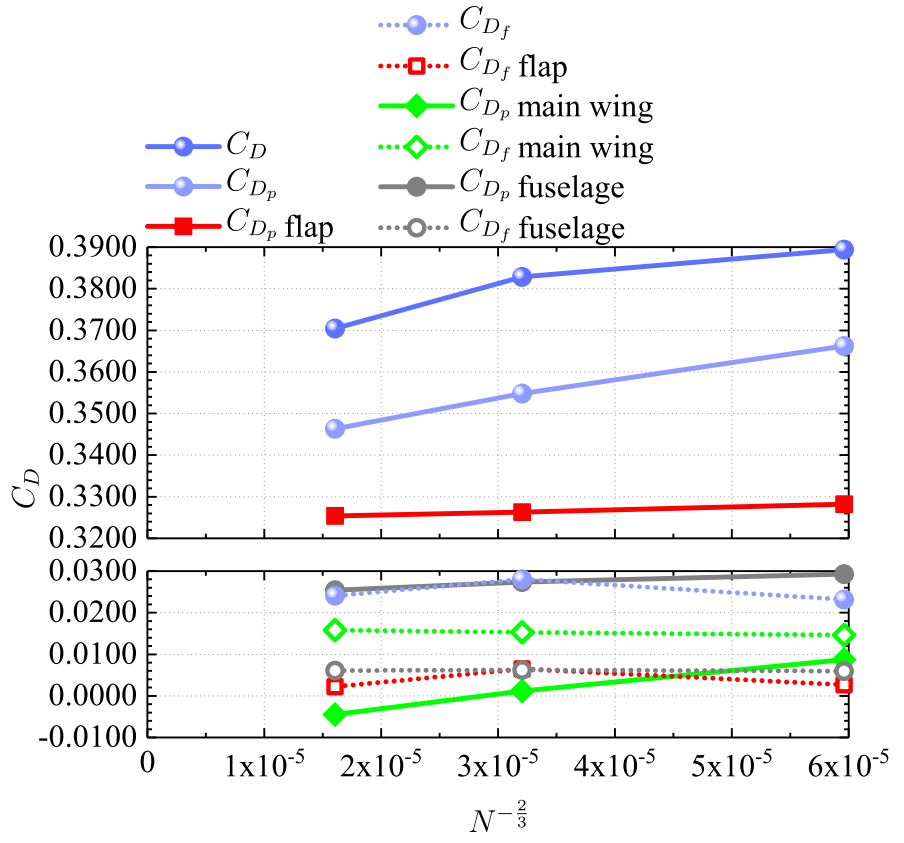

(b) $C_{D}$

Fig.5 Quantitative comparison of the mesh convergence by the Richardson's $N^{-2 / 3}$ function



(a) Coarse

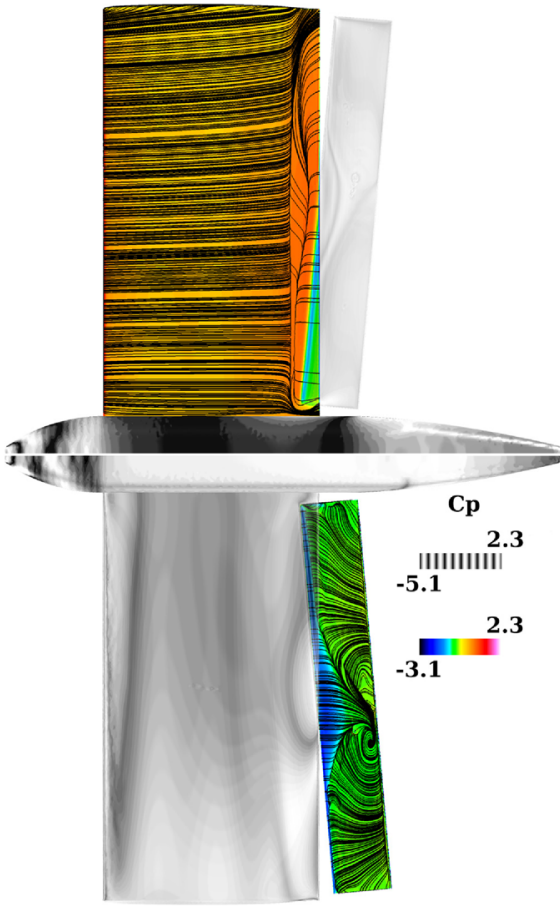

(b) Medium



(c) Fine
Fig. 6 The influence of mesh density on the CFD results using the $\psi$ of $-5.0^{\circ}$ configuration. The top half depicts the lower surface of the model; the bottom half illustrates the upper surface of it. The flap makes translucent representations in the upside so that we see the overlapping

By contrast, the Coarse mesh misses the separation of this region, according to Fig. 6a. The district does not induce the lift because the flow initially stagnates due to the separation- portion. Likewise, the wing represents semi-transparently in the downside. We draw the color contour of $C_{\mathrm{p}}$ with the lower limit value raised for emphasizing the contrast

however, the area in the Coarse mesh functions as a liftgenerating mechanism. Since the $C_{\mathrm{p}}$ of the site is low, the negative $C_{\mathrm{L}}$ transpires; the $C_{\mathrm{L}}$ by the Coarse mesh is lower 
than the actual $C_{\mathrm{L}}$. Conversely, the Coarse mesh appraises the $C_{\mathrm{L}}$ lower than it pragmatically is because of valuing the higher $C_{\mathrm{p}}$ around the flap upper surface.

\section{$3.2 C_{D}$}

Figure $5 \mathrm{~b}$ indicates that the pressure drag coefficient $C_{D_{\mathrm{p}}}$ is dominant over the friction drag coefficient $C_{\mathrm{D}_{\mathrm{f}}}$ for the total drag coefficient $C_{\mathrm{D}}$ because of

$C_{\mathrm{D}_{\mathrm{p}}}=\mathcal{O}\left(10^{-1}\right)>C_{\mathrm{D}_{\mathrm{f}}}=\mathcal{O}\left(10^{-2}\right)$.

So, the $C_{\mathrm{D}}$ gains monotonously due to the $\partial C_{\mathrm{D}_{\mathrm{p}}} / \partial N^{-2 / 3}$ (which is monotonous contrary to the non-monotonous $\left.\partial C_{\mathrm{D}_{\mathrm{f}}} / \partial N^{-2 / 3}\right)$. The finer the surface mesh, the smaller is $C_{\mathrm{D}}$. However, since there is a tradeoff between mesh density and computational cost, we will restrain the growth in mesh density as much as possible.

As stated above, the $C_{\mathrm{D}_{\mathrm{p}}}$ rules the $C_{\mathrm{D}}$, its proportion is 94.0\% in the Coarse mesh, $92.7 \%$ in the Medium mesh, and 93.5\% in the Fine mesh, respectively. Besides, the flap is the most influential component for the entire $C_{\mathrm{D}_{\mathrm{p}}}, 94.0 \%$ in the Coarse mesh, $92.0 \%$ in the Medium mesh, and $89.6 \%$ in the Fine mesh. Since we set $\alpha=0.0^{\circ}$ and chose the fuselage with as little impact on aerodynamic performances as possible, as a consequence the flap's $C_{\mathrm{D}_{\mathrm{p}}}$ controls the gross $C_{\mathrm{D}_{\mathrm{p}}}$.

Since we consider the flap's $C_{\mathrm{D}_{\mathrm{p}}}$ at the flap angle of $35.0^{\circ}$, the flap underside produces a high $C_{\mathrm{D}_{\mathrm{p}}}$. That is, the pressure on the flap undersurface rules the $C_{\mathrm{D}_{\mathrm{p}}}$ rather than that on its upper surface, where the Coarse mesh insufficiently captures the flow field. So, mesh dependency becomes small. Similarly, even when the CFD analyses grasp fluid states for the wing insufficiently, it does not affect the $C_{\mathrm{D}_{\mathrm{p}}}$. Thus, mesh convergence is linear. The variation in $\partial C_{\mathrm{D}_{\mathrm{p}}} / \partial N^{-2 / 3}$ for the wing and the flap depends on the difference in each component's projected area in the uniform flow direction. By contrast, the wetted area determines the magnitude of the $C_{D_{\mathrm{f}}}$. The rate of the separating area to the wetted area is more abundant in the flap than in the wing, so it is more susceptible to the mesh, resulting in a non-monotonic change in $\partial C_{\mathrm{D}_{\mathrm{f}}} / \partial N^{-2 / 3}$ of the flap.

\section{Results and discussion of yaw-wise rotational effect for the flap}

We analyze the $C_{\mathrm{L}}$ and the $C_{\mathrm{D}}$ at the five $\psi$ conditions with the Medium mesh density specified in the previous section. Figure 7 organizes the results. Since the computations suppose the landing state, we will center on the $C_{\mathrm{L}}$. Although Fig. 7 likewise shows the $C_{\mathrm{D}}$ for a reference, it is not an essential factor. We omit $L / D$ for the same reason.
Figure $7 \mathrm{a}$ indicates that the $C_{\mathrm{L}}$ values under two $\psi$ conditions gain more than that at $\psi=0.0^{\circ}$ as the reference. The flap at $\psi=-2.5^{\circ} /+2.5^{\circ}$ boosts the $C_{\mathrm{L}}$ by $+6.7 \% /+3.5 \%$ than that at $\psi=0.0^{\circ}$. By contrast, the flap at $\psi=-5.0^{\circ} /+5.0^{\circ}$ deteriorates the $C_{\mathrm{L}}$ by $-12.3 \% /-16.3 \%$ than the reference value. Figure 8 visualizes the flow structure to illuminate aerodynamic mechanisms by which the $C_{\mathrm{L}}$ varies due to $\psi$. Besides, Fig. 9 signifies the Mach contour on the $y-z$ plane at the wing trailing edge to examine how the gap accelerates the flow. In the following, we will divide into subsection and discuss physical mechanisms in each $\psi$ state using these data.

\section{$4.1 \psi=0.0^{\circ}$}

First, we discern the flow structure at $\psi=0.0^{\circ}$ shown in Fig. $8 \mathrm{c}$, which is the neutral reference condition. The flow on the flap upper surface separates at a similar chordwise location. At both the flap root and the flap tip, the vortices form while adhering to the flap sides. Thus, the separation occurs backward. Since the fuselage prevents the vortex from spreading near the flap root, it becomes weaker than that near the flap tip.

The separation on the flap upper surface near the flap root/tip occurs at the flap $60 \% / 64 \%$ chordwise location. In other regions, the separation line commences drawing a gentle curve with the $41 \%$ spanwise and $28 \%$ chordwise positions of the flap, as the onset. Since both flap sides produce vortices, the pressure declines; the velocity rises relative to the flap center. Moreover, the speed of the flap root side decelerates compared with the flap tip side owing to the fuselage. Figure $9 \mathrm{c}$ indeed indicates that the $41 \%$ spanwise station with the slowest flow velocity stipulates the separation distribution.

\section{$4.2 \psi= \pm 2.5^{\circ}$}

Figures $8 \mathrm{~b}$ and $\mathrm{d}$ declare that the separation structure is alike at between $\psi=-2.5^{\circ}$ and $+2.5^{\circ}$, despite the opposite yawwise rotational direction (namely, the gap broadness/narrow switches between the root and the tip sides). Outside the $28 \% / 21 \%$ spanwise place of the flap at $\psi=-2.5^{\circ} /+2.5^{\circ}$, the separation onset advances linearly on approaching the flap tip (near the flap tip, the separation onset retreats due to the influence of the flap tip vortex as well as at $\psi=0.0^{\circ}$ ).

Comparing the Mach contour shown in Fig. $9 \mathrm{~b}$ and d, the flow velocity on the wing upper surface near the wing trailing edge is faster than that at $\psi=0.0^{\circ}$ in Fig. $9 \mathrm{c}$ in the narrowed gap region. In other words, the separation onset on the flap retreats because the quickened air flows from the gap around the $41 \%$ spanwise location, so the area that the flap functions recovers. Then, $C_{\mathrm{L}}$ gains. 


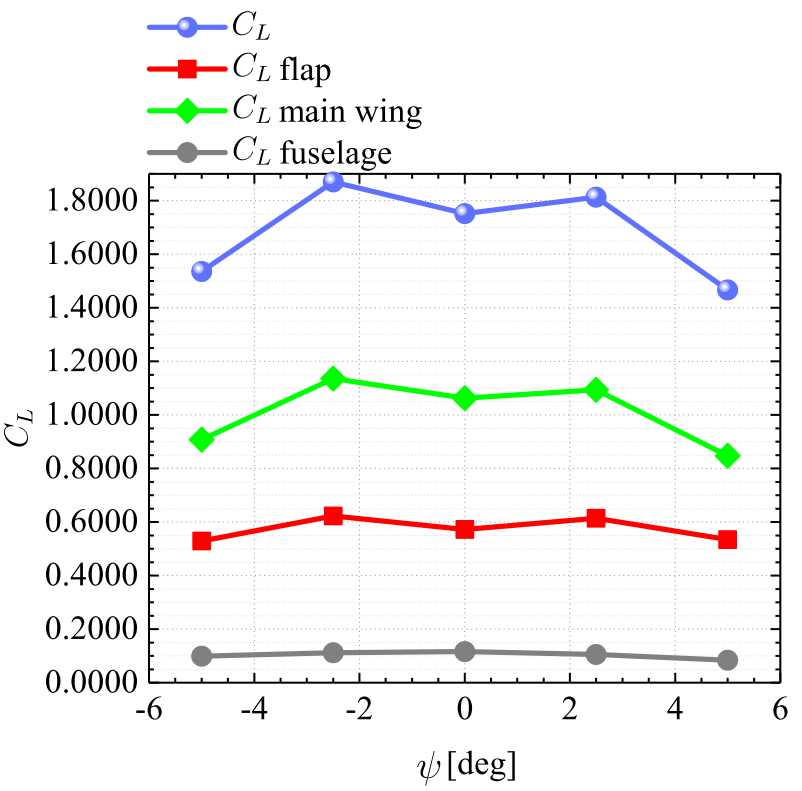

(a) $C_{L}$

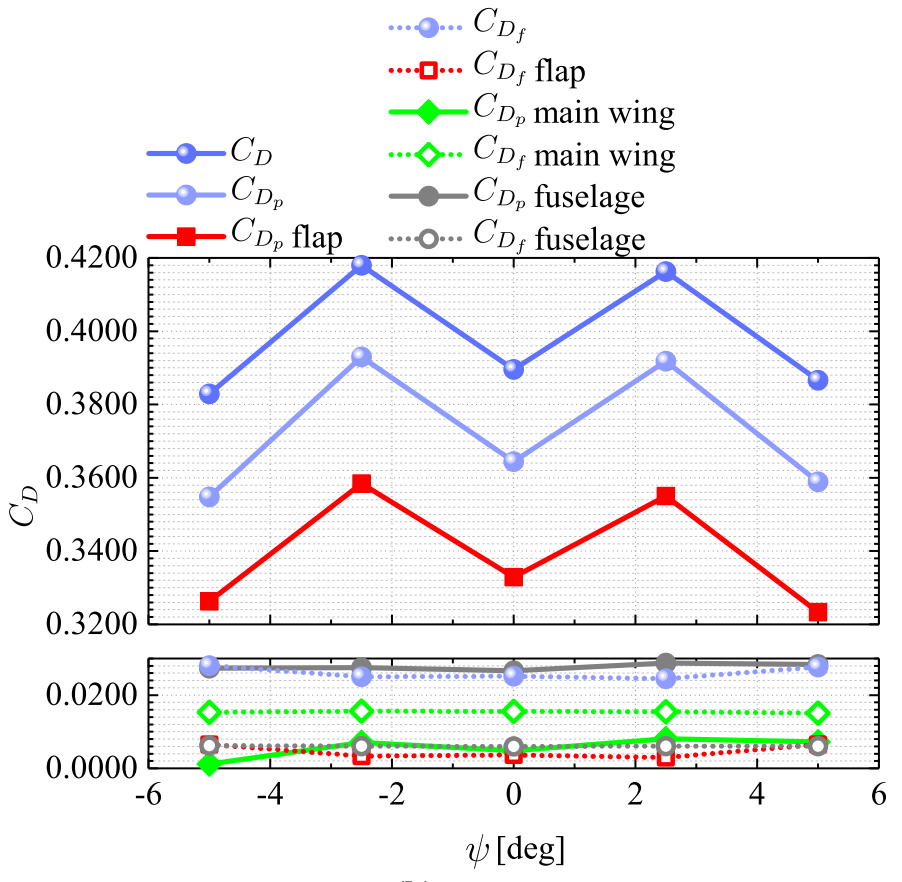

(b) $C_{D}$

Fig. 7 Comparison of the aerodynamic performance between each $\psi$ condition

However, the aspect of the velocity distribution is different between that at $\psi=-2.5^{\circ}$ and $+2.5^{\circ}$.

$-\psi=-2.5^{\circ}$ : the yaw-wise rotation expedites the flow from 12 to $39 \%$ of the gap spanwise location, as drawn in white in Fig. 9b. Outside 50\% gap spanwise scene, the flow speed is slower than that of $\psi=0.0^{\circ}$, which deteriorates the gap's role. Nevertheless, the flow speed on the wing upper surface grows over a wide range, and the separation line on the flap top surface retrogrades.

$-\psi=+2.5^{\circ}$ : the flow of the gap from 59 to $90 \%$ of the spanwise realm accelerates, as shown in white in Fig. 9d. The flow decelerates lower than that at $\psi=0.0^{\circ}$; the gap's function degrades inside the $50 \%$ spanwise position. The flow quickens up on the wing upper surface by the speed boost outside the $50 \%$ gap spanwise place. Hence, the separation line on the flap top surface retreats.

The results indicate that it is more effective to raise the $C_{\mathrm{L}}$ by accelerating the gap flow at a proper spot in the spanwise direction rather than by widening the speedup region of the gap excessively. The definitional constraint of linearly varying gaps endows the above results in this problem. Given the nonlinear separation structure in the spanwise direction, we presume that dividing the flap into numerous pieces in the spanwise direction and independently operating them is more effective in raising the $C_{\mathrm{L}}$.

\section{$4.3 \psi= \pm 5.0^{\circ}$}

Rotating $\psi$ to $\pm 5.0^{\circ}$ effects suppressing the separation on the flap, but does not influence raising the $C_{\mathrm{L}}$ because of the narrow spanwise scope of its impact, as shown in Fig. 8a and e. Although the local spanwise directional range eliminates the separation on the flap top surface, the gap does not serve the state of amelioration in other regions. Thus, the separation line shifts forward compared at $\psi=0.0^{\circ}$, resulting in lower $C_{\mathrm{L}}$ by the flap.

- Figure 9a and e declares that a functioning gap width becomes narrower than that at $\psi= \pm 2.5^{\circ}$. Thus, the rotation of $\psi=-5.0^{\circ}\left(+5.0^{\circ}\right)$ restricts the acceleration region to the range of $40-45 \%$ (48-52\%) of the flap spanwise location, and the maximum velocity is slower than that at $\psi=-2.5^{\circ}\left(\psi=+2.5^{\circ}\right)$.

- Since the flow velocity is also slower than that at $\psi=$ $0.0^{\circ}$ in the non-faster flow speed area $(<40 \% / 48 \%$ or $>45 \% / 52 \%$ spanwise realm at $\left.\psi=-5.0^{\circ} /+5.0^{\circ}\right)$, the separation onset on the flap upper surface moves forward rather than at $\psi=0.0^{\circ}$.

These flow structures do not have a crucial contrast between $\psi=-5.0^{\circ}$ and $\psi=+5.0^{\circ}$. As an outcome, the order of the $C_{\mathrm{L}}$ in each $\psi$ condition is

$C_{\mathrm{L}}^{\psi=-2.5}>C_{\mathrm{L}}^{\psi=+2.5}>C_{\mathrm{L}}^{\psi=0.0}>C_{\mathrm{L}}^{\psi=-5.0}>C_{\mathrm{L}}^{\psi=+5.0}$. 


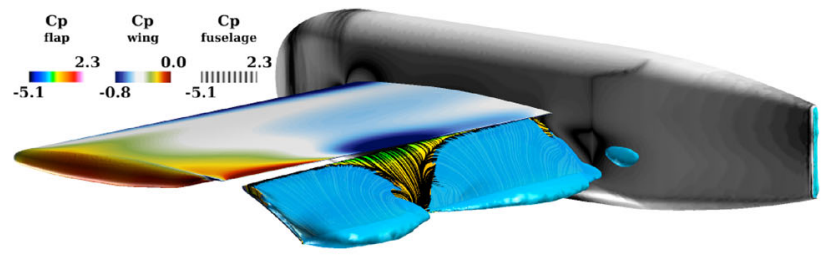

(a) $\psi=-5.0 \mathrm{deg}$

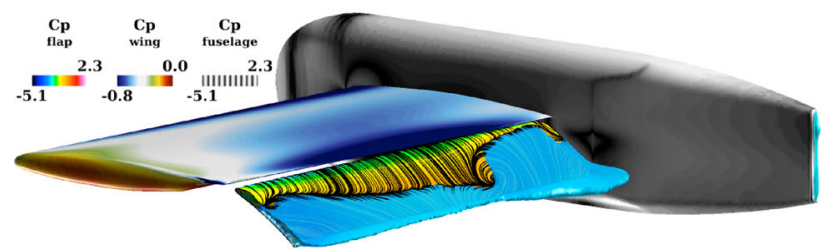

(b) $\psi=-2.5 \mathrm{deg}$

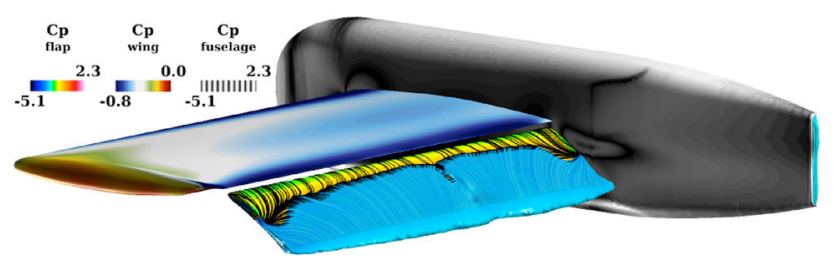

(c) $\psi=0.0 \mathrm{deg}$

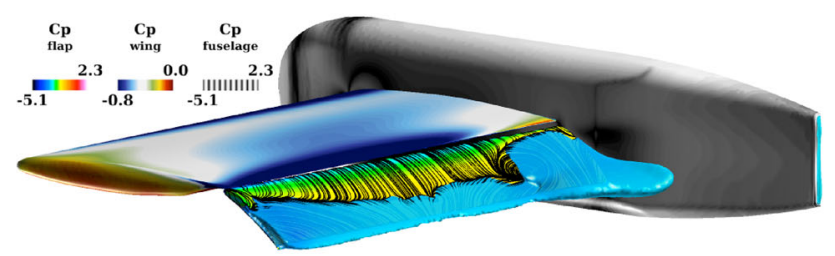

(d) $\psi=+2.5 \mathrm{deg}$

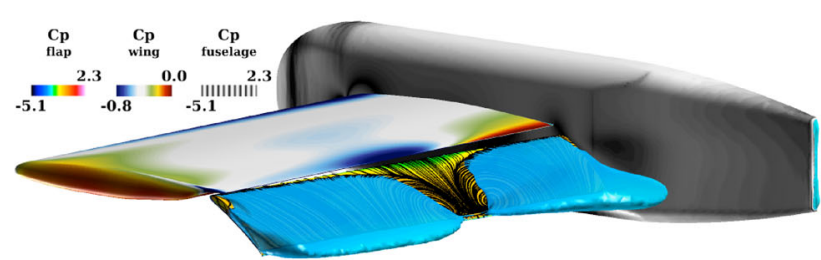

(e) $\psi=+5.0 \mathrm{deg}$

Fig. 8 Comparison of separation, $C_{\mathrm{p}}$ distribution, and streamlines on the flap between each $\psi$ condition

The flap with a small yaw-wise rotation achieves the $C_{\mathrm{L}}$ raise. In contrast, we cannot anticipate the $C_{\mathrm{L}}$ growth because of curtailing the fast velocity region, even if we set $|\psi|>5.0^{\circ}$.

\subsection{Influence for the $C_{p}$ distribution on the wing upper surface}

Figure 7 explains that the wing affects the total $C_{\mathrm{L}}$ more than the flap and that the $C_{\mathrm{L}}$ by the wing is more sensitive to $\psi$ than the $C_{\mathrm{L}}$ by the flap. Two regions of the low $C_{\mathrm{p}}$ arise on the wing upper surface in the chordwise direction, as shown in Fig. 8 (the $C_{\mathrm{p}}$ rises near the wingtip, as is the general situation). For the equal absolute value of $\psi$, the qualitative

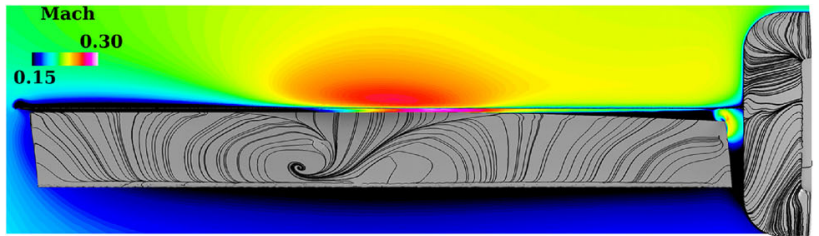

(a) $\psi=-5.0 \mathrm{deg}$

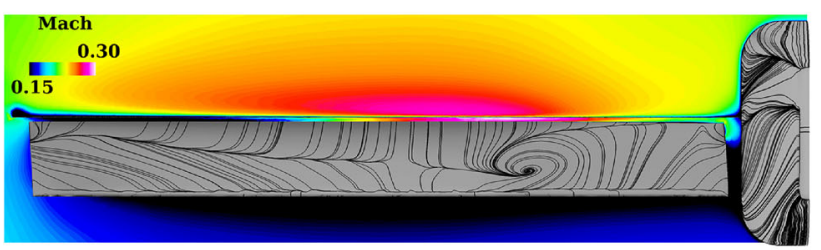

(b) $\psi=-2.5 \mathrm{deg}$

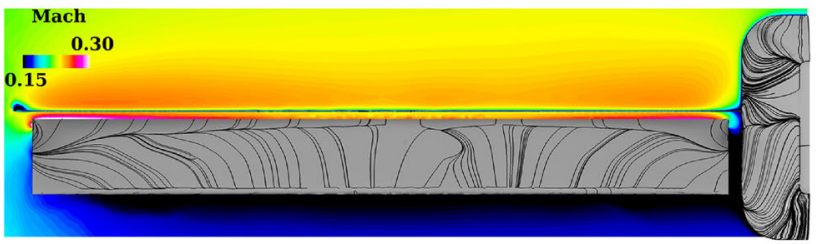

(c) $\psi=0.0 \mathrm{deg}$

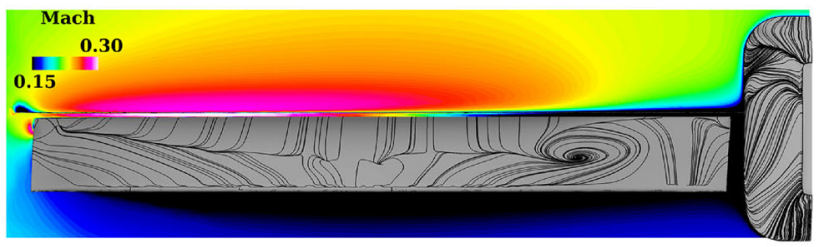

(d) $\psi=+2.5 \mathrm{deg}$

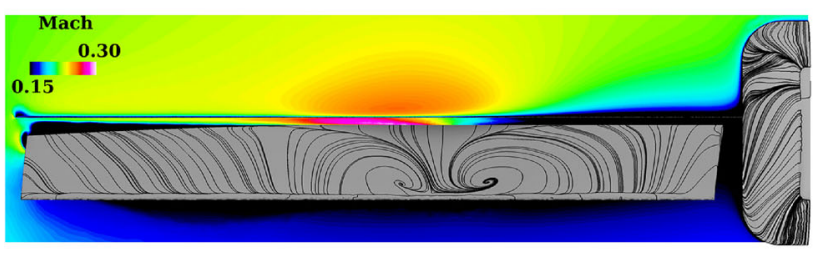

(e) $\psi=+5.0 \mathrm{deg}$

Fig. 9 Mach contour on the $y-z$ plane at the wing trailing edge. The viewpoint is behind the computational model

fluid structure is alike. However, the $C_{\mathrm{L}}$ obtained for- $\psi$ is higher due to the grown pressure around the wingtip. The following compiles the concrete aerodynamic diversity.

\subsection{1 $\Psi=0.0^{\circ}$}

Figure $8 \mathrm{c}$ indicates that roughly $40 \%$ to $70 \%$ chordwise location ("Region1") causes a $C_{\mathrm{p}}$ fall area; this region fades away in the vicinity of the wingtip. Near the wing trailing edge ("Region2"), the territory of the low $C_{\mathrm{p}}$ lengthens in a spanwise band-however, the $C_{\mathrm{p}}$ gains near the wingtip. 


\subsection{2 $\psi= \pm 2.5^{\circ}$}

The flow acceleration in a section of the gap yields $C_{\mathrm{p}}$ decreases in Region1 and Region2 shown in Fig. 8b and d. Also, it widens these regions rather than at $\psi=0.0^{\circ}$.

$-\psi=-2.5^{\circ}$ : as the gap at the wingtip side enlarges, the flow velocity around there declines, and the $C_{\mathrm{p}}$ intensifies. The effect of the gap spreading becomes small because the $C_{\mathrm{p}}$ grows by nature at $\psi=0.0^{\circ}$ due to the wingtip.

$-\psi=+2.5^{\circ}$ : as the gap on the wing root side widens, the flow velocity diminishes at that side, and the $C_{\mathrm{p}}$ rises. This result reduces the $C_{\mathrm{L}}$.

\subsection{3 $\psi= \pm 5.0^{\circ}$}

Figure 9a and e illustrates that a narrow portion of the gap accelerates the flow, but it is slower, and its range is narrower than the speed at $\psi=-2.5^{\circ}$. Hence, the $C_{\mathrm{p}}$ in Region 1 gains more than that at $\psi=0.0^{\circ}$, as shown in Fig. $8 \mathrm{a}$ and e. The whole $C_{\mathrm{p}}$ in Region 2 grows; the low- $C_{\mathrm{p}}$ region narrows.

The flow field at $\psi= \pm 5.0^{\circ}$ qualitatively resembles the state at $\psi= \pm 2.5^{\circ}$. Because the gap at $\psi= \pm 5.0^{\circ}$ enlarges more than that at $\psi= \pm 2.5^{\circ}$, the recovery rate of the $C_{\mathrm{p}}$ is also higher. Consequently, the $\psi= \pm 5.0^{\circ}$ raises the $C_{\mathrm{p}}$ more than at $\psi=0.0^{\circ}$, resulting in lower $C_{\mathrm{L}}$.

\subsection{Future studies}

This study considered merely one condition of $\alpha=0.0^{\circ}$ and $M=0.2$; the conclusion does not warrant the effect of the yaw-wise rotational flap under other conditions. Since the flow field around a slotted flap is extremely sensitive to the relative position between the wing and the flap originally, their optimal setting varies depending on flow field conditions. To substantiate the generality of the $C_{\mathrm{L}}$ growth mechanism aforementioned, we should optimize the aerodynamic performance with the flap angle, the gap, and the overlap as well as $\alpha$ and $M$ as design variables.

However, since the optimization is a massive task, the next point we should perform would be to examine the angle of attack effect. To suppress the repercussion of the wing in this study, we investigated under $\alpha=0.0^{\circ}$ condition. Even if $\alpha$ shifts, we obtain a comparable effect by quickening the flow around the appropriate location of the gap. However, the present results do not explain the influence of the yawwise rotation for the flap on the maximum lift coefficient $C_{\mathrm{L}_{\max }}$. We must scrutinize whether the yaw-wise rotational flap raises the $C_{\mathrm{L}_{\max }}$.

In the future, a comparison with wind tunnel test results is imperative to discuss aerodynamic performance errors on the $C_{\mathrm{L}}-C_{\mathrm{D}}$ curve and to bring the robustness to the $C_{\mathrm{L}}$ rise mechanism deduced by CFD. Thus, we are anticipating wind tunnel tests separately [3].

The outcome of this study ascertains that the yaw-wise rotational flap can ameliorate the $C_{\mathrm{L}}$. This study linearly adjusted the spanwise width of the gap between the wing and the flap by the yaw-wise rotation for the flap. However, if we remodel it nonlinearly, the effect of raising $C_{\mathrm{L}}$ can be augmented further. We will attempt it as another idea.

\section{Conclusion}

This study scrutinized the aerodynamic effect of adding a yaw-wise rotational degree of freedom to a single slotted flap of an airplane via computational fluid dynamic analyses for a simple model consisting of a fuselage, a wing, and a slotted flap. It will be natural that a gap between the wing and the flap corresponds to the 3D flow field formed on the flap upper surface. The spanwise gap admits placing nonlinearly by adding the degree of yaw-wise rotation to the flap with a degree of freedom of translation along flap tracks. Consequently, the flap set at an appropriate yaw-wise rotation angle had an effect of raising the lift. The fact resulted from a mechanism that the flow acceleration near the gap retreated the onset of the separation on the flap upper surface. The outcome indicates that a suitable gap setting to match each spanwise place must ameliorate the lift further.

Acknowledgements JSPS KAKENHI (JP16K00295) supported a part of the study. FieldView created whole CFD visual images, which Intelligent Light provides via its University Partners Program.

\section{Compliance with ethical standards}

Conflict of interest The authors declare that they have no conflict of interest.

Open Access This article is licensed under a Creative Commons Attribution 4.0 International License, which permits use, sharing, adaptation, distribution and reproduction in any medium or format, as long as you give appropriate credit to the original author(s) and the source, provide a link to the Creative Commons licence, and indicate if changes were made. The images or other third party material in this article are included in the article's Creative Commons licence, unless indicated otherwise in a credit line to the material. If material is not included in the article's Creative Commons licence and your intended use is not permitted by statutory regulation or exceeds the permitted use, you will need to obtain permission directly from the copyright holder. To view a copy of this licence, visit http://creativecomm ons.org/licenses/by/4.0/.

\section{References}

1. Baragona M, Boermans LMM, van Tooren MJL, Bijl H, Beukers A (2003) Bubble bursting and stall hysteresis on single-slotted flap high-lift configuration. J Aircr 14(7):1230-1237. https://doi.org/ $10.2514 / 2.2091$ 
2. Burg C (2005) Higher order variable extrapolation for unstructured finite volume RANS flow solvers. In: AIAA paper 2005-4999

3. Chiba K, Komatsu T, Kato H, Nakakita K (2020) Remote and feedback control of the flap angle in a wind tunnel test model by optical measurement. Aerospace 7(2):1-11. https://doi.org/10. 3390/aerospace7020011

4. Chiba K, Makino Y, Takatoya T (2012) Design-informatics approach for intimate configuration of silent supersonic technology demonstrator. J Aircr 49(5):1200-1211. https://doi.org/10.2514/1. $\mathrm{C} 031116$

5. van Dam CP (2002) The aerodynamic design of multi-element high-lift systems for transport airplanes. Prog Aerosp Sci 38(2):101-144. https://doi.org/10.1016/S0376-0421(02)00002-7

6. Filippone A (2010) Cruise altitude flexibility of jet transport aircraft. Aerosp Sci Technol 14(4):283-294. https://doi.org/10.1016/ j.ast.2010.01.003

7. Fincham JHS, Friswell MI (2015) Aerodynamic optimisation of a camber morphing aerofoil. Aerosp Sci Technol 43:245-255. https://doi.org/10.1016/j.ast.2015.02.023

8. Hahn AS (2010) Vehicle Sketch Pad: parametric geometry for conceptual aircraft design . In: AIAA Paper 2010-0657

9. Ito Y, Murayama M, Koike S, Yamamoto K, Nakakita K, Kusunose K (2019) Computational investigation of vertical stabilizer with vortex generators and dorsal fin. J Aircr 56(5):1833-1848. https:// doi.org/10.2514/1.C035301

10. Ito Y, Murayama M, Yamamoto K, Shih AM, Soni BK (2009) Efficient computational fluid dynamics evaluation of small device locations with automatic local remeshing. AIAA J 47(5):1270 1276. https://doi.org/10.2514/1.40875

11. Ito Y, Murayama M, Yamamoto K, Shih AM, Soni BK (2011) Efficient hybrid surface and volume mesh generation for viscous flow simulations. In: AIAA paper 2011-3539

12. Ito $\mathrm{Y}$, Nakahashi K (2002) Direct surface triangulation using stereolithography data. AIAA J 40(3):490-496. https://doi.org/10. 2514/2.1672

13. Ito Y, Nakahashi K (2002) Surface triangulation for polygonal models based on CAD data. Int J Numer Methods Fluids 39(1):75-96. https://doi.org/10.1002/fld.281

14. Ito Y, Nakahashi K (2004) Improvements in the reliability and quality of unstructured hybrid mesh generation. Int J Numer Methods Fluids 45(1):79-108. https://doi.org/10.1002/fld.669

15. Ito Y, Shih AM, Koomullil RP, Kasmai N, Jankun-Kelly M, Thompson D (2009) Solution adaptive mesh generation using feature-aligned embedded surface meshes. AIAA J 47(8):18791888. https://doi.org/10.2514/1.39378

16. Ito Y, Shih AM, Soni BK (2004) Reliable isotropic tetrahedral mesh generation based on an advancing front method. In: Proceedings of the 13th international meshing roundtable, Williamsburg, VA, pp 95-105

17. Ito Y, Shih AM, Soni BK (2009) Octree-based reasonable-quality hexahedral mesh generation using a new set of refinement templates. Int J Numer Methods Eng 77(13):1809-1833. https://doi. org/10.1002/nme. 2470

18. Ito Y, Shih AM, Soni BK (2011) Hybrid mesh generation with embedded surfaces using a multiple marching direction approach. Int J Numer Methods Fluids 67(1):1-7. https://doi.org/10.1002/fld. 1962

19. Ito Y, Shih AM, Soni BK (2011) Three dimensional automatic local remeshing for two or more hybrid meshes. Int J Numer Methods Fluids 66(12):1495-1505. https://doi.org/10.1002/fld.2324

20. Ito Y, Shih AM, Soni BK, Nakahashi K (2007) Multiple marching direction approach to generate high quality hybrid meshes. AIAA J 45(1):162-167. https://doi.org/10.2514/1.23260

21. Lin JC (2002) Review of research on low-profile vortex generators to control boundary-layer separation. Prog Aerosp Sci 38(4):389420. https://doi.org/10.1016/S0376-0421(02)00010-6
22. Little J, Samimy M (2010) High-lift airfoil separation with dielectric barrier discharge plasma actuation. AIAA J 48(12):2884-2898. https://doi.org/10.2514/1.J050452

23. Menter FR (1994) Two-equation eddy-viscosity turbulence models for engineering applications. AIAA J 32(8):1598-1605. https://doi. org/10.2514/3.12149

24. Murayama M, Yamamoto K (2008) Comparison study of drag prediction by structured and unstructured mesh method. J Aircr 45(3):799-822. https://doi.org/10.2514/1.31072

25. Murayama M, Yamamoto K, Ito Y, Hirai T, Tanaka K (2015) Japan Aerospace Exploration Agency studies for the second high-lift prediction workshop. J Aircr 52(4):1026-1041. https://doi.org/10. 2514/1.C033158

26. Murayama M, Yokokawa Y, Yamamoto K, Ura H, Imamura T, Hirai T, Ito Y, Kwak D, Kobayashi H, Shindo S (2014) Investigation of noise generation from bluff flap side-edge of a high-lift wing model. Trans Jpn Soc Aeronaut Sp Sci Aerosp Technol Jpn 12:9-16

27. Nakahashi K, Ito Y, Togashi F (2003) Some challenges of realistic flow simulations by unstructured grid CFD. Int J Numer Methods Fluids 43(6):769-783. https://doi.org/10.1002/fld.559

28. Obayashi S, Guruswamy GP (1994) Convergence acceleration of an aeroelastic Navier-Stokes solver. AIAA J 33(6):1134-1141. https://doi.org/10.2514/3.12533

29. Owens DB, McConnell JK, Brandon JM, Hall RM (2006) Transonic free-to-roll analysis of the F-35 (joint strike fighter) aircraft. J Aircr 43(3):608-615. https://doi.org/10.2514/1.16972

30. Panesar AS, Weaver PM (2012) Optimisation of blended bistable laminates for a morphing flap. Compos Struct 94(10):3092-3105. https://doi.org/10.1016/j.compstruct.2012.05.007

31. Petz R, Nitsche W (2007) Active separation control on the flap of a two-dimensional generic high-lift configuration. J Aircr 44(3):865-874. https://doi.org/10.2514/1.25425

32. Reckzeh D (2003) Aerodynamic design of the high-lift-wing for a megaliner aircraft. Aerosp Sci Technol 7:107-119. https://doi.org/ 10.1016/S1270-9638(02)00002-0

33. Richardson LF (1911) The approximate arithmetical solution by finite differences of physical problems including differential equations, with an application to the stresses in a masonry dam. Philos Trans Roy Soc A 210(459):307-357. https://doi.org/10.1098/rsta. 1911.0009

34. Rodriguez DL, Aftosmis MJ, Nemec M, Anderson GR (2016) Optimization of flexible wings with distributed flaps at off-design conditions. J Aircr 53(6):1731-1745. https://doi.org/10.2514/1. C033535

35. Rudolph PKC (1989) Airfoil flap assembly with flap track member. US Patent Re. 32,907.

36. Rumsey CL, Slotnick JP (2015) Overview and summary of the second AIAA high-lift prediction workshop. J Aircr 52(4):10061025. https://doi.org/10.2514/1.C032864

37. Rumsey CL, Slotnick JP, Long M, Stuever RA, Wayman TR (2011) Summary of the first AIAA CFD high-lift prediction workshop. J Aircr 48(6):2068-2079. https://doi.org/10.2514/1.C031447

38. Rumsey CL, Slotnick JP, Sclafani AJ (2018) Overview and summary of the third AIAA high-lift prediction workshop. J Aircr 56(2):621-644. https://doi.org/10.2514/1.C034940

39. Seele R, Tewes P, Woszidlo R, McVeigh MA, Lucas NJ, Wygnanski IJ (2009) Discrete sweeping jets as tools for improving the performance of the V-22. J Aircr 46(6):2098-2106. https://doi.org/ $10.2514 / 1.43663$

40. Sharov D, Nakahashi K (1998) Reordering of hybrid unstructured grids for Lower-Upper Symmetric Gauss-Seidel computations. AIAA J 36(3):484-486. https://doi.org/10.2514/2.392

41. Smith AMO (1975) High-lift aerodynamcis. J Aircr 12(6):501541. https://doi.org/10.2514/3.59830 
42. Takenaka K, Hatanaka K, Yamazaki W, Nakahashi K (2012) Multidisciplinary design exploration for a winglet. J Aircr 45(5):16011611. https://doi.org/10.2514/1.33031

43. Venkatakrishnan V (1995) Convergence to steady state solutions of the Euler equations on unstructured grids with limiters. J Comput Phys 118(1):120-130. https://doi.org/10.1006/jcph.1995.1084
44. Wenzinger CJ, Harris TA (1939) Wind-tunnel investigation of an NACA 23012 airfoil with various arrangements of slotted flaps. NACA-TR-664

45. Yokokawa Y, Murayama M, Ito T, Yamamoto K (2006) Experiment and CFD of a high-lift configuration civil transport aircraft model. In: AIAA paper 2006-3452 